This item was submitted to Loughborough's Research Repository by the author.

Items in Figshare are protected by copyright, with all rights reserved, unless otherwise indicated.

\title{
Acetaminophen ingestion improves muscle activation and performance during a 3-min all-out cycling test
}

PLEASE CITE THE PUBLISHED VERSION

https://doi.org/10.1139/apnm-2018-0506

\section{PUBLISHER}

(C) The Authors. Published by NRC Research Press

\section{VERSION}

AM (Accepted Manuscript)

\section{PUBLISHER STATEMENT}

This work is made available according to the conditions of the Creative Commons Attribution-NonCommercialNoDerivatives 4.0 International (CC BY-NC-ND 4.0) licence. Full details of this licence are available at: https://creativecommons.org/licenses/by-nc-nd/4.0/

\section{LICENCE}

CC BY-NC-ND 4.0

\section{REPOSITORY RECORD}

Morgan, Paul T., Anni Vanhatalo, Joanna L. Bowtell, Andrew M. Jones, and Stephen Bailey. 2019.

"Acetaminophen Ingestion Improves Muscle Activation and Performance During a 3-min All-out Cycling Test". figshare. https://hdl.handle.net/2134/35135. 


\section{Acetaminophen ingestion improves muscle activation and performance during a 3-min all-out cycling test}

Original investigation

Paul T. Morgan, Anni Vanhatalo, Joanna L. Bowtell, Andrew M. Jones and Stephen J. Bailey ${ }^{1}$

Department of Sport and Health Sciences, College of Life and Environmental Sciences, University of Exeter, St. Luke’s Campus, Heavitree Road, Exeter, EX1 2LU, UK.

\section{Address for Correspondence:}

Andrew M Jones, Ph.D.

Department of Sport and Health Sciences, College of Life and Environmental Sciences, University of Exeter, St. Luke’s Campus, Heavitree Road, Exeter, EX1 2LU, UK.

Tel: 01392722815

E-mail1: A.M.Jones@exeter.ac.uk

E-mail2: P.T.Morgan@exeter.ac.uk

E-mail ${ }^{3}$ : J.Bowtell@exeter.ac.uk

E-mail ${ }^{4}$ A.Vanhatalo@exeter.ac.uk

E-mail ${ }^{5}$ : S.Bailey2@lboro.ac.uk

${ }^{1}$ Present address for Stephen J Bailey: School of Sport, Exercise and Health Sciences, Loughborough University, Ashby Road, Loughborough, Leicestershire LE11 3TU

Running title: Acetaminophen and power-duration relationship

Abstract word count: 246 words

Text-only word count: 4767 words

Disclosure of funding: This research was not supported by any funding body external to University of Exeter 


\section{ABSTRACT}

2 Purpose: Acute acetaminophen (ACT) ingestion has been shown to enhance cycling time-

trial performance. The purpose of this study was to assess whether ACT ingestion enhances muscle activation and critical power (CP) during maximal cycling exercise. Methods: Sixteen active male participants completed two 3-min all-out tests against a fixed resistance on an electronically-braked cycle ergometer 60 minutes following ingestion of $1 \mathrm{~g}$ ACT or placebo (maltodextrin, PL). CP was estimated as the mean power output over the final $30 \mathrm{~s}$ of the test and $\mathrm{W}^{\prime}$ (the curvature constant of the power-duration relationship) was estimated as the work done above CP. The femoral nerve was stimulated every $30 \mathrm{~s}$ to measure membrane excitability (M-wave) and surface electromyography (EMGRMS) was recorded continuously to infer muscle activation. Results: Compared to PL, ACT ingestion increased CP (ACT: 297 \pm 32 vs PL: $288 \pm 31 \mathrm{~W}, P<0.001$ ) and total work done (ACT: $66.4 \pm 6.5$ vs PL: $65.4 \pm 6.4$ $\mathrm{kJ}, P=0.03$ ) without impacting $\mathrm{W}^{\prime}(\mathrm{ACT}: 13.1 \pm 2.9$ vs PL: $13.6 \pm 2.4 \mathrm{~kJ}, P=0.19)$ or the Mwave amplitude $(P=0.66)$ during the 3-min all-out cycling test. Normalized EMGRMS amplitude declined throughout the 3-min protocol in both PL and ACT conditions; however, the decline in EMGRMS was attenuated in the ACT condition, with the EMGRMs amplitude being greater compared to PL over the last $60 \mathrm{~s}$ of the test $(P=0.04)$. Conclusion: These findings indicate that acute ACT ingestion might increase performance and CP during maximal cycling exercise by enhancing muscle activation.

Key words: Analgesic; critical power; electromyography; muscle activation; neuromuscular fatigue; exercise performance 
Fatigue is a complex, multi-factorial process that is linked to perturbations within the central nervous system and the contracting skeletal muscles (Enoka \& Duchateau, 2008; Hureau et al. 2016). Recent studies suggest that fatigue development may be related, at least in part, to pain sensation (Astokorki \& Mauger 2017a; Astokorki \& Mauger 2017b; O’Leary et al. 2017). Acetaminophen (ACT) is a commonly used medicine for general pain relief. Ingestion of ACT lowers pain sensation through inhibiting the cyclooxygenase enzymes, which stimulate nociceptor discharge through the synthesis of prostaglandins (Graham et al. 2013; Jóźwiak-Bębenista \& Nowak, 2014), and modulating serotoninergic, opioid and cannabinoid pathways (Graham et al. 2013; Pickering et al. 2006, 2008). Acute ACT ingestion has been shown to enhance endurance exercise performance consistent with the notion that interventions which can modulate pain sensation have the potential to influence exercise performance (Foster et al. 2014; Mauger et al. 2010, Morgan et al. 2018). Indeed, similar to the effects of caffeine (O’Connor et al. 2004), Mauger et al. (2010) and Foster et al. (2014) have both previously reported enhanced exercise performance and/or work output at a given level of muscle pain following acute ACT ingestion. These results suggest that ACT reduces pain at a given absolute work rate and/or permits a higher work rate for an equivalent pain sensation.

In a recent study, Morgan et al. (2018) reported an attenuated decline in skeletal muscle electromyography (EMG) amplitude, reflective of an increase in muscle activation, and an increased critical torque during a maximal intermittent single-leg knee extensor test following ACT ingestion. During cycling exercise, the power equivalent of the critical torque, the critical power (CP), represents an important threshold for oxidative metabolic control and exercise tolerance (Jones et al. 2010; Vanhatalo et al. 2011). Indeed, CP, which is the 
asymptote of the hyperbolic relationship between power output and time to exhaustion, reflects the highest work rate that can be sustained without a progressive loss of intramuscular and systemic homeostasis (Black et al. 2016; Poole et al. 1988; Poole et al. 1990; Vanhatalo et al. 2016), and interacts with the curvature constant of this relationship, W', to define exercise tolerance within the severe exercise intensity domain (Jones et al. 2010; Vanhatalo et al. 2011). Since CP is linked to muscle activation and neuromuscular fatigue development during exercise, as inferred from EMG responses (Burnley et al. 2012), and since ACT ingestion can concomitantly influence EMG responses and the critical torque (Morgan et al., 2018), ACT might also enhance CP by modulating aspects of central fatigue development during large muscle mass exercise. This potential blunting in central fatigue development could be mediated by inhibition of nociceptor sensitising prostaglandins (Graham et al. 2013; Jóźwiak-Bębenista \& Nowak, 2014) and/or enhanced corticospinal excitability (Mauger \& Hopker, 2013) permitting an increased CP and thus improved endurance exercise performance.

Although the improvement in cycling performance that has been reported following ACT ingestion (Foster et al. 2014; Mauger et al. 2010) may also be linked to enhanced neuromuscular function and a higher $\mathrm{CP}$, as observed during single leg exercise (Morgan et al. 2018), the exercise modality and the volume of skeletal muscle mass engaged are known to influence the degree of neuromuscular and peripheral fatigue development. Specifically, greater peripheral fatigue development has been observed at the same relative intensity during knee-extensor exercise compared to cycling exercise (Rossman et al. 2012, 2014). Therefore, the mechanisms underpinning the potential ergogenic effect of ACT on larger muscle mass exercise such as cycling, which is more relevant for sports performance, requires further research. 
The purpose of the present study was, therefore, to assess the effect of acute ACT ingestion

on neuromuscular fatigue development and its potential underlying mechanisms during large muscle mass exercise. We tested the hypotheses that, compared to placebo, acute consumption of $1 \mathrm{~g}$ ACT would increase total work done, CP and muscle activation during a 3-min all-out cycling test.

MATERIALS AND METHODS

Participants

Sixteen trained male cyclists (mean \pm SD: age $29 \pm 9$ y, height $1.79 \pm 0.07 \mathrm{~m}$, body mass 77 $\pm 8 \mathrm{~kg}, \dot{\mathrm{V}} \mathrm{O}_{2 \text { peak }} 60.8 \pm 7.0 \mathrm{ml} \cdot \mathrm{kg}^{-1} \cdot \mathrm{min}^{-1}$, range: $52-77 \mathrm{ml} \cdot \mathrm{kg}^{-1} \cdot \mathrm{min}^{-1}$ ) provided written informed consent to participate in the present study, which was approved by the local Ethics Committee (Sport and Health Sciences, University of Exeter). All subjects participated in local cycling competitions. Trained individuals were selected as it has been shown that endurance training influences pain tolerance (O’Leary et al. 2017). After being informed of the experimental procedures and associated risks, all participants completed a medical health questionnaire, which was checked by a medical doctor, to ensure it was safe to consume ACT prior to performing exhaustive exercise. The questionnaire incorporated questions pertaining to: known allergies to medications, current intake of medication and prior use of ACT as well as any history of illnesses, cigarette use, alcohol consumption, illegal drug use and chronic illnesses (personal and family history). None of the participants had a history of motor and/or neurological disorders or frequent chronic ingestion of pain relief medication (i.e. ACT, nonsteroidal anti-inflammatory medication etc.). Participants were also advised to avoid ingestion of pain relief medication over the duration of the study and were provided with a 
rested and fully hydrated state, at least 3 h post-prandial, and to avoid strenuous exercise, and consumption of caffeine and alcohol in the $24 \mathrm{~h}$ prior to each testing session.

\section{Experimental Design}

Participants visited the laboratory on 5 occasions over a 5- to 6-week period with all tests conducted at a similar time of day $( \pm 90 \mathrm{~min})$. All tests were conducted on an electronically braked cycle ergometer (Lode Excalibur Sport, Groningen, Netherlands). On the first laboratory visit, participants performed a ramp-incremental cycling test for the determination of the linear factor (as described below), gas exchange threshold (GET), peak aerobic power output and the peak oxygen uptake ( $\dot{\mathrm{V}}_{2 \text { peak }}$ ). During this initial laboratory visit, the seat and handlebar positions were adjusted for comfort and replicated for all tests. The second and third laboratory visits were used to familiarise participants to the measurements and experimental protocol as described below. During these visits (i.e. visits 2-3), participants completed a 3-min all-out cycling test to ensure the coefficient of variation for work done and CP between visits was $<1 \%$ and that the criteria to ensure a valid test were fulfilled (Jones et al. 2010). For each 3-min test, achievement of $\dot{\mathrm{V}} \mathrm{O}_{2 \text { peak }}(>95 \%)$, as verified by the $\dot{\mathrm{V}} \mathrm{O}_{2 \text { peak }}$ achieved during the ramp incremental ramp tests, was an obligatory criterion for a valid test. In the one instance where these criteria were not fulfilled, the participant completed a further familiarisation trial prior to commencing the experimental trials. During these sessions, the settings and placement of EMG and peripheral nerve stimulation electrodes were recorded for each subject as a reference for electrode placement in subsequent experimental trials (see below for further details). These trials were not included in the subsequent data analysis. Participants then performed the fatiguing protocol under two experimental conditions: placebo (PL) and ACT. Experimental sessions were separated by 3-7 days. 
124 All trials (visits 1-5) started with a standardised warm-up routine (10 min at 100-150 W, corresponding to $<90 \%$ GET, followed by 5 min of passive rest) and testing of the optimal EMG electrode (for recording muscle activation), anode and cathode placement and stimulation intensity for peripheral nerve stimulation. Single peripheral nerve stimulation pulses were manually triggered at rest to determine the characteristics of the M-wave response to supra-maximal nerve stimulation. Neuromuscular function was assessed pre-, during- and post-trial (<10 s) as described below.

The experimental protocol comprised a 3-min period of unloaded pedalling at the participant's preferred cadence, followed by a 3-min all-out sprint, 60 min following ingestion of either PL (1 g maltodextrin) or $1 \mathrm{~g}$ ACT (visits 4-5). This timing was selected to coincide with the attainment of the peak plasma [ACT] concentration (Anderson et al. 2008). The placebo was made from dextrose powder inserted into gelatine capsules designed to have an identical appearance and weight to ACT capsules but without the analgesic and antipyretic effects. The order of trials for visits 4 and 5 were administered in a double-blind, randomised fashion using a counter-balanced cross-over experimental design. The 3-min all-out cycling protocol used in this study replicated the procedures described previously by Vanhatalo et al. (2007, 2008). The fixed resistance for the all-out sprint was set using the linear mode of the ergometer such that on reaching their preferred cadence, the participants would achieve a power output equivalent to $50 \%$ of the difference between GET and $\dot{\mathrm{VO}}_{2 \text { peak }}$ (linear factor $=$ $50 \% \Delta$ power output/preferred cadence ${ }^{2}$ ). 
Throughout all laboratory tests, participants wore a mask connected to an impeller turbine transducer assembly (Cortex Metalyzer, Cortex, Leipzig, Germany). Inspired and expired gas volume and concentration signals were continuously sampled at $100 \mathrm{~Hz}$. The analyser was calibrated before each test with gases of known concentration $\left(\mathrm{O}_{2} 15 \%, \mathrm{CO}_{2} 4.5 \%\right)$, and a calibration syringe of known volume (3-L; Hans Rudolph, KS).

\section{Electromyography}

Neuromuscular function was assessed pre-, during- and immediately post each of the trials. Pre- and post-trial neuromuscular function was tested with the participant cycling at 80 RPM with a low resistance $(20 \mathrm{~W})$ as described below. Surface EMG activity was measured from $m$. vastus lateralis, $m$. vastus medialis, $m$. rectus femoris and $m$. biceps femoris muscles of the right leg to continuously record muscle activity during exercise using active bipolar bar electrodes with a single differential configuration (DE2.1, DelSys Inc, Boston, MA, USA). Bipolar electrodes were positioned over the muscle belly parallel to the longitudinal axis of each muscle (SENIAM guidelines). The placement of electrodes was considered optimal on achieving the largest and most reproducible M-wave signal from the $m$.vastus lateralis and $m$.vastus medialis whilst noting minimal activity in the $m$.bicep femoris. Placement of electrodes was optimised during each laboratory visit. Double-sided adhesive tape and a hypoallergenic medical tape were used to ensure the EMG sensor stability for recording electrodes. The skin area underneath each EMG electrode was shaved, then exfoliated and cleaned with alcohol to minimise the skin impedance. The EMG signal was pre-amplified (1000 x), band-pass filtered (20-450 Hz, Bagnoli-8, DelSys Inc, Boston, MA, USA), and then transferred to a computer with a sampling frequency of $2 \mathrm{kHz}$. EMG data were recorded continuously and digitised synchronously with 16 bit resolution via an $\mathrm{A} / \mathrm{D}$ converter $( \pm 5 \mathrm{~V}$ range, CED 1401 power, Cambridge, UK). EMG was average rectified using the root mean 
square method (EMG $\mathrm{RMS}_{\text {). }} \mathrm{EMG}_{\mathrm{RMS}}$ throughout the trial was then normalised to the EMG signal during the first $30 \mathrm{~s}$ of the 3-min test to provide a percentage of the maximal signal. Finally, EMG $\mathrm{RMS}_{\mathrm{R}}$ was normalised to the local (closest) standardised M-wave amplitude and presented as a percentage of the maximal signal. In addition, M-wave amplitude was normalised by pre-exercise, resting values, and presented as a percentage. This method of normalizing the EMG trace to the M-wave may enable a more accurate assessment of changes in muscle activation that are likely occurring upstream of the neuromuscular junction (i.e. spinal and/or supraspinal in origin). The ground electrode was placed over the patella of the right leg.

\section{Peripheral Nerve Stimulation}

Electrical stimulation was applied using a constant current stimulator (Digitimer Stimulator DS7AH, Digitimer, UK). Initially, the crank angle at which peripheral nerve stimulation was to be delivered during the trials was determined for each subject as described by Black et al. (2017) and as performed by Sidhu et al. (2012). Stimulations were delivered at the identified crank angle specific to each trial (62 $\pm 7^{\circ}$ relative to full knee extension, $\left.180^{\circ}\right)$ to align with maximal EMG $\mathrm{RMS}_{\mathrm{R}}$ amplitude. A custom written sequencer script triggered 3 single stimulations, independently, with at least 1 and up to 10 pedal revolutions between stimuli. During the 3-min cycling test, these stimulations were delivered every $30 \mathrm{~s}$. M-waves were elicited in $m$.vastus lateralis and $m$.vastus medialis by supramaximal percutaneous electrical stimulation of the femoral nerve (200 $\mu$ s duration), approximately $3-5 \mathrm{~cm}$ below the inguinal ligament in the femoral triangle. The cathode was systematically moved vertically and horizontally and the amplitude of the muscle action potential (i.e. M-wave) was monitored to identify the optimal position of the cathode for attaining maximal peak-to-peak M-wave $\left(\mathrm{M}_{\max }\right)$ amplitude. To determine the stimulation intensity, single stimuli were delivered in 20 
mA step-wise increments from $100 \mathrm{~mA}$ until a plateau (i.e. $\mathrm{M}_{\max }$ ) in the $\mathrm{M}$-wave was observed. A supramaximal pulse of $130 \% \mathrm{M}_{\max }$ current (Burke, 2002; Goodall et al. 2010; Neyroud et al. 2014) was applied during the exercise tests (mean stimulation intensity: $251 \pm$ $48 \mathrm{~mA}$ ). The procedures for optimal electrode placement and stimulation intensity were completed during each laboratory visit (visits 2-5).

\section{Data Analysis}

Data were analysed using a custom written script developed in Spike2 software (CED, Cambridge, UK). CP was estimated as the mean power output over the final $30 \mathrm{~s}$ of the test, and the $\mathrm{W}^{\prime}$ was estimated as the work done above the CP (Vanhatalo et al. 2007, 2008). Peak $\dot{\mathrm{V}}_{2}$ was determined as the highest 15 -s interval (i.e. $\dot{\mathrm{VO}}_{2 \text { peak}}$ ). Total work was calculated as the area under the power-time curve. Peak power output attained in the 3-min test was defined as the maximal 1-s interval. The changes in power output, $\mathbf{M}_{\mathrm{Max}}$ and $\mathrm{EMG} \mathrm{GMS}_{\mathrm{R}}$, were used to quantify neuromuscular fatigue development and changes in muscle activation. All neuromuscular parameters and power output were averaged across the protocol into $6 \times 30$-s bin averages. Estimates of $\mathrm{CP}$ and $\mathrm{W}^{\prime}$ were also used to predict the time taken to complete a range of total work done (W) targets (50, 75, 100, 125, 150, 175, 200, 225, 250, 500, 750, $1000 \mathrm{~kJ}$ ) as previously described (Kelly et al. 2013).

\section{Statistics}

Paired-samples $t$-tests were used to compare the $\mathrm{CP}, \mathrm{W}^{\prime}$, total work done and cardiorespiratory responses between ACT and PL conditions. In addition, paired samples ttests were used to assess parameters of neuromuscular function at task end between trials (i.e. 
$\mathrm{M}_{\max }$ and $\left.\mathrm{EMG}_{\mathrm{RMS}}\right)$. The profiles of power output, M-wave amplitude and EMG $\mathrm{RMS}_{\text {before, }}$ during and after the 3-min test were analysed using two-way ANOVAs (time $\times$ condition) with repeated measures (using 30 s averages; i.e. 6 time points) between PL and ACT. A twoway repeated-measures ANOVA was also used to assess differences in predicted performance times. Where the ANOVA revealed a significant interaction effect, post-hoc comparisons were completed using a Bonferroni correction. A Pearson's product moment correlation coefficient was used to determine the relationship between the change in EMG amplitude and the change in power production between conditions. A one-way ANOVA was used to assess differences in $\dot{\mathrm{V}} \mathrm{O}_{2 \text { peak }}$ obtained during the incremental ramp test and both 3min trials (PL and ACT). To assess the possibility of an order effect of trials, a paired samples t-test was conducted on total work done for visits 4 and 5 . For calculation of effect size, partial eta squared $\left(\eta^{2}\right)$ was used for omnibus tests. Cohen's $d$ was used to calculate the effect size for paired $t$-tests and post-hoc comparisons. All statistical tests were performed both on \% change and raw data. Where sphericity was violated, a Greenhouse Geisser correction factor was used. For all tests, results were considered statistically significant when $P<0.05$. Data are presented as mean $\pm \mathrm{SD}$, unless otherwise indicated. All statistical analyses were conducted using IBM SPSS Statistics version 24.

\section{RESULTS}

Mean $\dot{\mathrm{V}}{ }_{2 \text { peak }}$ measured in the ramp incremental test was $4.50 \pm 0.41 \mathrm{~L} \cdot \mathrm{min}^{-1}(61 \pm 6$ $\mathrm{ml} \cdot \mathrm{kg}^{-1} \cdot \mathrm{min}^{-1}$ ) and the peak aerobic power output was $393 \pm 29 \mathrm{~W}$. The GET occurred at $1.98 \pm 0.26 \mathrm{~L} \cdot \mathrm{min}^{-1}$ and $152 \pm 22 \mathrm{~W}$. The $\dot{\mathrm{V}}{ }_{2 \text { peak }}$ achieved during the 3-min test following PL $\left(4.51 \pm 0.59 \mathrm{~L} \cdot \mathrm{min}^{-1}, 60 \pm 7 \mathrm{ml} \cdot \mathrm{kg}^{-1} \cdot \mathrm{min}^{-1}\right)$ and ACT ingestion $\left(4.53 \pm 0.57 \mathrm{~L} \cdot \mathrm{min}^{-1}, 61\right.$ $\pm 8 \mathrm{ml} \cdot \mathrm{kg}^{-1} \cdot \mathrm{min}^{-1}$ ) were not significantly different to the values achieved during the ramp 247 incremental test $(P=0.77)$. 
3-min all-out cycling test

The $\dot{\mathrm{VO}}_{2}$ profile during the 3-min test for PL and ACT conditions is shown in figure 1 (panel

A). In addition, the mean power output profile for all participants (and differences in CP) during the 3-min all-out cycling test is shown in figure 1 (panel B) for the PL and ACT conditions. Panel C represents changes to power output throughout the duration of the 3-min test in all trials and is provided in 30-s averages. During the PL trial, power output declined from $820 \pm 139 \mathrm{~W}$ during the first $5 \mathrm{~s}$ of the test to $288 \pm 31 \mathrm{~W}$ during the last $30 \mathrm{~s}$ of the 3min test $\left(P<0.0001, \eta^{2}=0.99\right.$; table 1$)$. However, during the ACT trial, power output declined from $838 \pm 127 \mathrm{~W}$ during the first $5 \mathrm{~s}$ of the test to $297 \pm 32 \mathrm{~W}$ during the final $30 \mathrm{~s}$ of the test (table 1). There was a significant interaction effect (time $\times$ condition; $P=0.04, \eta^{2}=0.26$ ) with the mean power output in the 3-min cycling test being greater in ACT (368 $\pm 36 \mathrm{~W}$ ) compared to PL (363 $\pm 36 \mathrm{~W}, P=0.007, d=0.13)$. CP (ACT: $297 \pm 32 \mathrm{~W}$ vs. PL: $288 \pm 31 \mathrm{~W}$, $P<0.0001, d=0.28$ ) and total work done (ACT: $66.4 \pm 6.5 \mathrm{~kJ}$ vs. $65.4 \pm 6.4 \mathrm{~kJ} ; P=0.03$, $d=0.15$ ) was higher with ACT compared to PL (table 1; figure 2). However, there was no difference in peak power output (ACT: $838 \pm 127 \mathrm{~W}$ vs. PL: $820 \pm 139 \mathrm{~W}, P=0.10, d=0.16$ ) or $\mathrm{W}^{\prime}(\mathrm{ACT}: 13.1 \pm 2.9$ vs. PL: $13.6 \pm 2.4 \mathrm{~kJ} ; P=0.19, d=0.20)$ during the 3-min cycling test between conditions. No order effect was observed between visit 4 and visit 5 for total work done (Visit 4: $65.8 \pm 6.5 \mathrm{~kJ}$ vs. Visit 5: $66.0 \pm 6.4 \mathrm{~kJ} ; P=0.75, d=0.03$ ).

When the $\mathrm{CP}$ and $\mathrm{W}^{\prime}$ were combined to predict the time required to complete fixed work targets between 50 and $1000 \mathrm{~kJ}$, using equation 1, the ANOVA revealed a main effect by condition $\left(P<0.0001, \eta^{2}=0.56\right)$ and an interaction effect $\left(P<0.0001, \eta^{2}=0.86\right.$, table 2$)$. Posthoc analysis revealed that the performance times were lower in the ACT condition compared 
with the PL condition for all time-trials with the exception of the two shortest (i.e. 50 and 75 $\mathrm{kJ})$, with the improvement ranging from 1.1\% (100 kJ) to 3.0\% (1000 kJ).

\section{Neuromuscular Function}

From pre to post exercise, there was a main effect for time on M-wave amplitude in the m.vastus lateralis ( $P=0.003, \eta^{2}=0.29$, figure 3 ), which declined as the protocol progressed. However, there was no main effect by condition $\left(P=0.66, \eta^{2}=0.01\right)$ or time $\times$ condition interaction effect $\left(P=0.70, \eta^{2}=0.03\right)$. $\mathrm{EMG}_{\mathrm{RMS}}$ in the $m$.vastus lateralis decreased from $94 \pm$ $4 \%$ over the first $30 \mathrm{~s}$ to $54 \pm 17 \%$ over the final $30 \mathrm{~s}$ of the 3 -min all-out test in the PL trial $\left(P<0.0001, \eta^{2}=0.50\right.$; figure 4). However, this decline in $\mathrm{EMG}_{\mathrm{RMS}}$ was attenuated following ACT ingestion (from $92 \pm 5$ over the first $30 \mathrm{~s}$ to $72 \pm 18 \%$ over the final $30 \mathrm{~s}$ of the 3 -min all-out test), with there being a time $\times$ condition interaction effect $\left(P=0.04, \eta^{2}=0.23\right)$. Posthoc analysis revealed EMGRMs was elevated at $150 \mathrm{~s}(P=0.02, d=0.84)$ and $180 \mathrm{~s}(P=0.001$, $d=1.31$ ) in ACT compared to PL (figure 4). There was a significant positive correlation between the change in EMG amplitude and the change in power production over the last $30 \mathrm{~s}$ of exercise between conditions ( $r=0.88, P=0.04$, figure 5$)$.

\section{DISCUSSION}

Consistent with our hypotheses, the principal original findings of this study were that acute ACT ingestion enhanced total work done and $\mathrm{CP}$, and attenuated the decline in EMG amplitude, in trained individuals during a 3-min all-out cycling test. The ACT-induced increase in $\mathrm{CP}$ was predicted to translate into a $1-3 \%$ reduction in the time required to complete a range of target work cycling trials $(100-1000 \mathrm{~kJ})$. The results of this study provide some insight into the mechanisms by which ACT ingestion is ergogenic during large muscle 
mass exercise and suggest that enhanced performance following ACT ingestion is attributable, at least in part, to increases in CP and muscle activation.

\section{Power-duration relationship}

300 Our finding of an increase in total work done following acute ACT ingestion in the 3-min allout cycling test is consistent with previous observations of enhanced exercise performance following acute ACT ingestion of similar doses (1-1.5 g; Foster et al. 2014; Mauger et al. 2010, Morgan et al. 2018). In the present study, neuromuscular fatigue development was assessed during the completion of a 3-min all-out cycling test to offer insight into the potential underlying mechanisms for the ergogenic effects of ACT ingestion. Consistent with our previous finding of a $4 \%$ increase in critical torque when utilising a single-limb kneeextension model (Morgan et al. 2018), CP achieved during a 3-min all-out cycling test was improved by $\sim 3 \%$ following the acute ingestion of ACT in the present study. Moreover, and consistent with our previous findings (Morgan et al. 2018), $\mathrm{W}^{\prime}$ was not altered following ACT ingestion in the current study.

The potential practical significance of the 3\% improvement in CP becomes clear when applied to an exercise performance scenario. An important practical application of the CP is that this parameter, in conjunction with $\mathrm{W}^{\prime}$, can be used to robustly predict cycling TT performance (Black et al. 2014, 2017; Burnley et al. 2012; Chidnok et al. 2013; Florence \& Weir, 1997; Skiba et al. 2012; Smith et al. 1999). Accordingly, the influence of a given intervention on $\mathrm{CP}$ and $\mathrm{W}^{\prime}$ can be used to predict the effect that that intervention might have on endurance exercise performance. For example, although Kelly et al. (2013) reported no statistically significant increase in either CP $(+1.4 \%)$ or $\mathrm{W}^{\prime}(+8.4 \%)$ following dietary nitrate supplementation, when the combined effect on these parameters was integrated, an 
improvement of $2-3 \%$ in cycling time-trial performance was predicted. Similarly, in the current study, endurance performance was predicted to be improved by $~ 1-3 \%$ following acute ACT ingestion in the work trial simulations ( 5-60 min). Since this magnitude of performance enhancement following acute ACT ingestion exceeds $0.6 \%$, which is suggested to be the smallest 'worthwhile' improvement in road TT cycling (Paton \& Hopkins, 2006), our results suggest that acute ACT ingestion may enable a practically meaningful improvement in endurance exercise performance. It should also be noted that, although we did not directly assess the effect of acute ACT ingestion on cycling TT performance in the current study, the predicted $1-3 \%$ is similar to the empirically demonstrated $1.8 \%$ improvement in 10-mile cycling TT performance reported previously (Mauger et al. 2010).

Interestingly, improvements in exercise performance with acute ACT ingestion have been reported in trained participants in both the current study and in previous studies (Mauger et al. 2010) despite evidence that endurance training increases pain tolerance (Jones et al. 2014; O’Leary et al. 2017) such that trained individuals are more likely to have a greater tolerance to pain (Janal et al. 1994; Tesarz et al. 2013). However, it should be stressed that, although the current and previous studies support an ergogenic effect of acute ACT consumption (Foster et al. 2014; Mauger et al. 2010, 2014; Morgan et al. 2018), regular ACT use, or exceeding a single dose of $1 \mathrm{~g}$, is not recommended given the hepatotoxicity of ACT (Graham et al. 2013).

\section{Neuromuscular function}

In addition to influencing the degree of muscle metabolic perturbation and the trajectory of the $\dot{\mathrm{VO}}_{2}$ slow component during exercise (Jones et al. 2008, 2010; Poole et al. 1988; Vanhatalo et al. 2011), CP is linked to muscle activation characteristics during exercise, as 
inferred from EMG responses, and is a critical threshold for neuromuscular fatigue

347 development (Burnley et al. 2012). Indeed, concomitant with our observation of an increased

348 CP in the current study, the decline in EMG amplitude during the 3-min all-out test was 349 attenuated in ACT compared to PL. These findings are strikingly similar to our recent study, 350 which reported a blunted decline in the EMG amplitude and an increased critical torque during a 5-min maximal intermittent single-legged knee extension exercise task (Morgan et al. 2018). Together, these results suggest that improved maintenance of muscle activation contributes to the elevated CP and total work done following ACT ingestion. However, the blunting of neuromuscular fatigue development following ACT ingestion was not accompanied by improvements in peripheral muscle excitability, as inferred from measurements of M-wave amplitude between the ACT and PL trials, suggesting that this alteration occurred due to mechanisms upstream of the neuromuscular junction.

Our results support the notion that the ergogenic effect of ACT is principally mediated centrally (Anderson, 2008; Graham et al. 2013; Smith, 2009; Toussaint et al. 2010). However, while we are not aware of any evidence to suggest that ACT might influence peripheral muscle excitability (Mauger \& Hopker, 2013), or that interventions aimed at reducing inflammation improve performance during whole body exercise (i.e. Cleak, \& Eston. 1992; Da Silva et al. 2015; Nosaka \& Clarkson, 1996; Tokmakdis et al. 2003), we cannot exclude that peripheral factors that were not assessed in the current study, such as inflammation and/or alterations to muscle metabolism, may have contributed to the ergogenic effect of ACT. Moreover, due to the nature of cycling exercise, it is technically challenging to directly test cortical alterations via changes to voluntary activation using the interpolated twitch technique (Doyle-Baker et al. 2017). 
Whilst we have previously investigated the contribution of central and peripheral factors to the improved performance following ACT ingestion in a small muscle mass model (Morgan et al. 2018), the mechanisms underpinning fatigue development, and therefore ACT's potential ergogenic effect, could differ for large muscle mass exercise (Rossman et al. 2012, 2014). We observed a strong correlation between the change in end-exercise EMG $\mathrm{RMS}_{\text {s }}$ and the change in power output (i.e. CP) within the last $30 \mathrm{~s}$ of the 3 -min cycling test $(r=0.88)$ following ACT ingestion compared to placebo. However, the change in $\mathrm{EMG}_{\mathrm{RMS}}$ was much larger than the change in CP. Although the mechanisms for this effect remain to be defined, this observation is in agreement, with Felippe et al. (2018). Specifically, these authors reported that, compared to placebo, caffeine ingestion increased mean power output by $\sim 4 \%$ during a 4-km cycling test, resulting in a $2 \%$ reduction in time to complete the $4-\mathrm{km}$ distance, alongside a $~ 17 \%$ increase in muscle recruitment (as inferred by EMG).

It is possible that, through lowering pain sensation (Foster et al. 2014; Mauger et al. 2010), ACT might have permitted the development of, and/or tolerance to, a greater degree of intramuscular metabolic perturbation beyond that required to evoke a 'critical' threshold of peripheral fatigue, thereby permitting improved exercise performance (Blain et al. 2016). Alternatively, since the effects of ACT are believed to be largely centrally mediated (Anderson, 2008; Graham et al. 2013; Smith, 2009; Toussaint et al. 2010), it is possible that ACT ingestion attenuated the development of central fatigue. A blunting in central fatigue development following ACT ingestion would be expected to permit enhanced central motor output, possibly through a reduction in inhibitory feedback via cyclooxygenase inhibition and a resultant decline in the synthesis of prostaglandins. 
The higher $\mathrm{EMG}_{\mathrm{RMS}}$ during the latter stages of the 3-min all-out cycling test observed following ACT ingestion may have been a consequence of enhanced corticospinal excitability (Mauger \& Hopker, 2013). Greater corticospinal excitability following ACT ingestion, as inferred from a greater motor-evoked potential in the study of Mauger \& Hopker (2013), may be linked to enhanced firing of motor units, and increased spinal excitability, as has been reported with caffeine consumption (i.e. Kalmar \& Cafarelli, 2004; Walton et al. 2003). Together, these effects on motor cortical and/or spinal excitability may explain the enhanced muscle activation and the subsequent greater amount of work performed with ACT ingestion in the current study. However, since cortical and peripheral contributions to fatigue development were not directly tested in this study, further research is required to resolve the underlying mechanisms for the ACT-mediated enhancement in muscle activation and performance during maximal exercise.

In conclusion, acute ACT ingestion increased total work done during a 3-min all-out cycling test in agreement with earlier reports of an ergogenic effect of ACT ingestion on cycling performance. The improved performance in the 3-min all-out test was accompanied by an increase in CP and better preservation of the EMG amplitude during the latter stages of the protocol. When the ACT-induced increase in CP was used to predict the effects of acute ACT ingestion on cycling performance, the estimated 1-3\% improvement was in line with previous experimental observations. Therefore, our results extend previous reports by revealing that ACT ingestion improves performance concomitant with enhanced CP and muscle activation during a 3-min all-out cycling test. These observations provide insight into the ergogenic effect of ACT ingestion during large muscle mass exercise. 


\section{Conflict of interest}

419 The authors report no conflict of interest in the publication of this research

420

\section{Acknowledgements}

422 This research was not sponsored by any funding body external to University of Exeter. The 423 authors report no conflict of interest in the publication of this research. The results are 424 presented clearly, honestly, and without fabrication, falsification or inappropriate data 425 manipulation.

426

\section{Author contribution}

428 P.T. Morgan, A. Vanhatalo, A.M. Jones and S.J. Bailey conceived and designed the research. 429 P.T. Morgan conducted all experiments. J.L. Bowtell provided assistance with pilot testing 430 prior to experimental data collection as well supporting data analysis. P.T. Morgan wrote the 431 manuscript. J.L. Bowtell, A. Vanhatalo, A.M. Jones and S.J. Bailey helped supervise the 432 project throughout. All authors contributed to the interpretation of results and read, edited and approved the manuscript. 


\section{References}

435

436

437

438

439

440

441

442

443

444

445

446

447

448

449

450

451

452

453

454

455

456

457

458

Anderson, B.J. 2008. Paracetamol (Acetaminophen): mechanisms of action. Paediatr Anaesth. 18: 915-921.

Astokorki, A.H.Y., and Mauger, A.R. 2017a. Tolerance of exercise-induced pain at a fixed rating of perceived exertion predicts time trial cycling performance. Scand. J. Med. Sci. Sports. 27(3): 309-317.

Astokorki, A.H.Y., and Mauger, A.R. 2017b. Transcutaneous electrical nerve stimulation reduces exercise-induced perceived pain and improves endurance exercise performance. Eur. J. Appl. Physiol. 117(3): 483-492.

Black, M.I., Durant, J., Jones, A.M., and Vanhatalo, A. 2014. Critical power derived from a 3 min all-out test predicts 16.1-km road time-trial performance. Eur. J. Sport Sci. 14: 217-223.

Black, M.I., Jones, A.M., Blackwell, J.R., Bailey, S.J., Wylie, L.J., McDonagh, S.T.J., Thompson, C., Kelly, J., Sumners, P., Mileva, K.N., et al. 2017. Muscle metabolic and neuromuscular determinants of fatigue during cycling in different exercise intensity domains. J. Appl. Physiol. 122(3): 446-459.

Blain, G.M., Mangum, T.S., Sidhu, S.K., Weavil, J.C., Hureau, T.J., Jessop, J.E., Bledsoe, A.D., Richardson, R.S., and Amann, M. 2016. Group III/IV muscle afferents limit the intramuscular metabolic perturbation during whole body exercise in humans. J. Physiol. 594(18): 5303-5315. 
459 Burke, D. 2002. Effects of activity on axonal excitability: implications for motor control

460

461

462

463

464

465

466

467

468

469

470

471

472

473

474

475

476

477

478

479

480

481

482

483

studies. Adv. Exp. Med. Biol. 508: 33-37.

Burnley, M., Vanhatalo, A., and Jones, A.M. 2012. Distinct profiles of neuromuscular fatigue during muscle contractions below and above the critical torque in humans. J. Appl. Physiol. 113: $215-223$.

Chidnok, W., Fulford, J., Bailey, S.J., DiMenna, F.J., Skiba, P.F., Vanhatalo, A., and Jones, A.M. 2013. Muscle metabolic determinants of exercise tolerance following exhaustion: relationship to the “critical power.” J. Appl. Physiol. 115(2): 243-250.

Cleak, M.J., and Eston, R.G. 1992. Muscle soreness, swelling, stiffness and strength loss after intense eccentric exercise. Br. J. Sports Med. 26: 267-272.

Da Silva, E., Pinto, R.S., Cadore, E.L., and Kruel, L.F. 2015. Nonsteroidal anti-inflammatory drug use and endurance during running in male long-distance runners. J. Athl. Train. 50: 295-302.

Enoka, R.M., and Duchateau, J. 2008. Muscle fatigue: what, why and how it influences muscle function. J. Physiol. 586: 11-23.

Felippe, L.C., Ferreira, G.A., Learsi, S.K., Boari, D., Bertuzzi, R., and Lima-Silva, A.E. 2018. Caffeine increases both total work performed above critical power and peripheral fatigue during a 4-km cycling time trial. J. Appl. Physiol. 24(6): 1491-1501. 
Florence, S., and Weir, J.P. 1997. Relationship of critical velocity to marathon running performance. Eur. J. Appl. Physiol. Occ. Physiol. 75(3): 274-8.

486

Foster, J., Taylor, L., Chrismas, B.C., Watkins, S.L., and Mauger, A.R. 2014. The influence of acetaminophen on repeated sprint cycling performance. Eur. J. Appl. Physiol. 114: 41-48.

489

490

Goodall, S., Ross, E.Z., and Romer, L.M. 2010. Effect of graded hypoxia on supraspinal 491 contributions to fatigue with unilateral knee-extensor contractions. J. Appl. Physiol. 109(6): 1842-51.

493

494

Graham, G.G., Davies, M.J., Day, R.O., Mohamudally, A., and Scott, K.F. 2013. The modern 495 pharmacology of paracetamol: therapeutic actions, mechanism of action, metabolism, toxicity 496 and recent pharmacological findings. Inflammopharmacology, 21: 201-232.

497

498

499

Hureau, T.J., Romer, L.M., and Amann, M. 2016. The 'sensory tolerance limit': A hypothetical construct determining exercise performance? Eur. J. Sport Sci. 7: 1-12.

500

501

502

503

Janal, M.N., Glusman, M., Kuhl, J.P., and Clark, W.C. 1994. Are runners stoical? An examination of pain sensitivity in habitual runners and normally active controls. Pain, 58(1): 109-116.

504

505

Jones, A.M., Vanhatalo, A., Burnley, M., Morton, R.H., and Poole, D.C. 2010. Critical 506 power: Implications for determination of VO2max and exercise tolerance. Med. Sci. Sports Exerc. 42(10): 1876-1890. 
Jones, A.M., Wilkerson, D.P., DiMenna, F., Fulford, J., and Poole, D.C. 2008. Muscle metabolic responses to exercise above and below the "critical power" assessed using ${ }^{31} \mathrm{P}$ MRS. Am. J. Physiol. Regul. Integr. Comp. Physiol. 294: 585-593.

Kalmar, J.M., and Cafarelli, E. 2004. "Central Fatigue and Transcranial Magnetic Stimulation: Effect of Caffeine and the Confound of Peripheral Transmission Failure.” J. Neuros Methods, 138(1-2): 15-26.

Kelly, J., Vanhatalo, A., Wilkerson, D.P., Wylie, L.J., and Jones, A.M. 2013. Effects of nitrate on the power-duration relationship for severe-intensity exercise. Med. Sci. Sports Exerc. 45(9): 1798-1806.

Mauger, A.R., and Hopker, J.G. 2013. The effect of acetaminophen ingestion on corticospinal excitability. Can. J. Physiol. Pharmacol. 91: 187-189.

Mauger, A.R., Jones, A.M., and Williams, C.A. 2010. Influence of acetaminophen on performance during time trial cycling. J. Appl. Physiol. 108: 98-104.

Mauger, A.R., Taylor, L., Harding, C., Wright, B., Foster, J., and Castle, P.C. 2014. Acute acetaminophen (paracetamol) ingestion improves time to exhaustion during exercise in the heat. Exp. Physiol. 99: 164-171.

Morgan, P.T., Bowtell, J.L., Vanhatalo, A., Jones, A.M., and Bailey, S.J. 2018. Acute acetaminophen ingestion improves performance and muscle activation during maximal intermittent knee extensor exercise. Eur. J. Appl. Physiol. 118(3): 595-605. 
535 Neyroud, D., Vallotton, A., Millet, G.Y., Kayser, B., and Place, N. 2014. The effect of 536 muscle fatigue on stimulus intensity requirements for central and peripheral fatigue 537 quantification. Eur. J. Appl. Physiol. 114(1): 205-15.

539 Nosaka, K., and Clarkson, P.M. 1996. Changes in indicators of inflammation after eccentric exercise of the elbow flexors. Med. Sci. Sports Exerc. 28: 953-961.

541

O’Connor, P.J., Motl, R.W., Broglio, S.P., and Ely, M.R. 2004. "Dose-Dependent Effect of

Caffeine on Reducing Leg Muscle Pain during Cycling Exercise Is Unrelated to Systolic Blood Pressure.” Pain, 109(3): 291-98.

545

O’Leary, T.J., Collett, J., Howells, K., and Morris, M.G. 2017. High but not moderate547 intensity endurance training increases pain tolerance: a randomised trial. Eur. J. Appl. Physiol. 117(11): 2201-2210.

549

550

Paton, C.D., and Hopkins, W.G. 2006. Variation in performance of elite cyclists from race to 551 race. Eur. J. Sport Sci. 6(1): 25-31.

552

Pickering, G., Estève, V., Loriot, M.A., Eschalier, A., and Dubray, C. 2008. Acetaminophen reinforces descending inhibitory pain pathways. Clin. Pharmacol. Ther. 84: 47-51.

555

Pickering, G., Loriot, M.A., Libert, F., Eschalier, A., Beaune, P., and Dubray, C. 2006. Analgesic effect of acetaminophen in humans: first evidence of a central serotonergic mechanism. Clin. Pharmacol. Ther. 79: 371-378. 
560 Poole, D.C., Burnley, M., Vanhatalo, A., Rossiter, H.B., and Jones, A.M. 2016. Critical

561 power: An important fatigue threshold in exercise physiology. Med. Sci. Sports Exerc. 562 48(11): 2320-2334.

563

564 Poole, D.C., Ward, S.A., Gardner, G.W., and Whipp, B.J. 1988. Metabolic and respiratory 565 profile of the upper limit for prolonged exercise in man. Erg. 31: 1265-79.

566

567 Rossman, M.J., Garten, R.S., Venturelli, M., Amann, M., and Richardson, R.S. 2014. The 568 role of active muscle mass in determining the magnitude of peripheral fatigue during 569 dynamic exercise. Am. J. Physiol. Regul. Integr. Comp. Physiol. 306(12): 934-940.

570

571 Rossman, M.J., Venturelli, M., Mcdaniel, J., Amann, M., and Richardson, R.S. 2012. Muscle

572 mass and peripheral fatigue: A potential role for afferent feedback? Acta Physiologica, 573 206(4): 242-250.

574

575 Sidhu, S.K., Cresswell, A.G., and Carroll, T.J. 2012. Motor cortex excitability does not 576 increase during sustained cycling exercise to volitional exhaustion. J. Appl. Physiol. 113(3):

577 401-409.

578

579 Skiba, P.F., Chidnok, W., Vanhatalo, A., and Jones, A. 2012. Modeling the expenditure and 580 reconstitution of work capacity above critical power. Med. Sci. Sports Exerc. 44(8): 1526581 1532. 
583

584

585

586

587

588

589

590

591

592

593

594

595

596

597

598

599

600

601

602

603

604

605

606

607

Smith, H.S. 2009. Potential analgesic mechanisms of acetaminophen. Pain Physician, 12: 269-80.

Smith, J.C., Dangelmaier, B.S., and Hill, D.W. 1999. Critical power is related to cycling time trial performance. Int. J. Sports Med. 20: 374-378.

Tesarz, J., Gerhardt, A., Schommer, K., Treede, R.D., and Eich, W. 2013. Alterations in endogenous pain modulation in endurance athletes: An experimental study using quantitative sensory testing and the cold-pressor task. Pain, 154(7): 1022-1029.

Toussaint, K., Yang, X.C., Zielinski, M.A., Reigle, K.L., Sacavage, S.D., Nagar, S., and Raffa, R.B. 2010. What do we (not) know about how paracetamol (acetaminophen) works? J Clin Pharm. Ther. 35: 617-638.

Vanhatalo, A., Black, M.I., DiMenna, F.J., Blackwell, J.R., Schmidt, J.F., Thompson, C., Wylie, L.J., Mohr, M., Bangsbo, J., Krustrup, P., et al. 2016. The mechanistic bases of the power-time relationship: muscle metabolic responses and relationships to muscle fibre type. J. Physiol. 594(15): 4407-4423.

Vanhatalo, A., Doust, J.H., and Burnley, M. 2008. A 3 min all-out cycling test is sensitive to a change in critical power. Med. Sci. Sports Exerc. 40(9): 1693-1699.

Vanhatalo, A., Doust, J.H., and Burnley, M. 2007. Determination of critical power using a 3min all-out cycling test. Med. Sci. Sports Exerc. 39(3): 548-555. 
608 Vanhatalo, A., Jones, A.M., and Burnley, M. 2011. Application of critical power in sport. Int. 609 J. Sports Physiol. Perform. 6(1): 128-136.

610

611 Walton, C., Kalmar, J., and Cafarelli, E. 2003. Caffeine increases spinal excitability in 612 humans. Muscle Nerve, 28: 359-36 


\section{Figure captions}

614 Figure 1

615 Group mean $\pm \mathrm{SE} \dot{\mathrm{V}} \mathrm{O}_{2}$ during acetaminophen (ACT, filled circles) and placebo (PL, clear 616 circles) is presented in panel $\mathrm{A}$. The dashed line represents the $\dot{\mathrm{V}} \mathrm{O}_{2 \text { peak }}$ attained in the 617 incremental ramp test. Panel B illustrates the mean \pm SE power output profile during the 3618 min maximal cycling protocol for placebo (clear circles) and acetaminophen (filled circles) 619 trials derived from $15 \mathrm{~s}$ averages. Note that after attainment of peak power output a few seconds into the test, power output falls over the first $~ 90-120$ s before reaching stable values

621 (the end-test power output; i.e. CP). CP is significantly elevated in the last $30 \mathrm{~s}$ of the ACT 622 condition. Significant changes to power output over time (derived from $30 \mathrm{~s}$ averages) throughout the 3-min cycling test for both ACT and PL conditions are shown in panel C. *Significantly different from PL (i.e. main effect of condition); ${ }^{\mathrm{a}}$ significantly different from $30 \mathrm{~s}$; ${ }^{\mathrm{b}}$ significantly different from $60 \mathrm{~s}$; ${ }^{\mathrm{c}}$ significantly different from $90 \mathrm{~s}$; ${ }^{\mathrm{d}}$ significantly different from $120 \mathrm{~s}$ (main effect of time, $P<0.05$ ).

627

Figure 2

629 Group mean total work done in the placebo (PL) and acetaminophen (ACT) conditions are shown in the open and closed bars, respectively (Panel A). Individual responses in the PL and ACT conditions are shown by the open circles and linked with dashed lines. * Significantly different from PL $(P<0.05)$. Panel B represents the group mean critical power $(\mathrm{CP})$ in the PL and ACT conditions in the open and closed bars, respectively. Individual responses in the PL and ACT conditions are shown by the open circles and linked with dashed lines. 
637 M-wave amplitude responses in the m.vastus lateralis during the 3-min cycling test for placebo (clear circles) and acetaminophen (filled circles) trials. Mean \pm SE M-wave responses are presented in panel A with the M-wave response from a representative 640 individual presented in panel B, for PL (grey line) and ACT (black line), for the first 30 and final $30 \mathrm{~s}$, respectively of the 3-min protocol. ${ }^{\mathrm{a}}$ significantly different from baseline; bsignificantly different from $30 \mathrm{~s}(P<0.05)$.

643

\section{Figure 4}

645 Surface electromyography (EMG) responses (expressed relative to M-wave amplitude) in the m.vastus lateralis during the 3-min cycling test for placebo (clear circles) and acetaminophen

647 (filled circles) trials. Mean \pm SE EMG responses are presented in panel A with the EMG response from a representative individual presented in panel B, for PL (grey line) and ACT 649 (black line), for the first 30 and final 30 s, respectively of the 3-min protocol. *Significantly 650 different from placebo; ${ }^{a}$ significantly different from $30 \mathrm{~s}$; ${ }^{b}$ significantly different from $60 \mathrm{~s}$; 651 ' significantly different from $90 \mathrm{~s}$; ${ }^{d}$ significantly different from $120 \mathrm{~s}$; ${ }^{e}$ significantly different 652 from $150 \mathrm{~s}(P<0.05)$.

653

\section{$654 \quad$ Figure 5}

655 Correlation between the change in electromyography amplitude (EMG, \%) and the change in 656 critical power (CP) between conditions (acetaminophen and placebo). The solid line 657 represents the line of best fit. 